\title{
Clopidogrel-AsSOCiATEd Thrombotic ThrombocytopeniC Purpura: A Case Report and Brief Review
}

Benjamin C. Creelan, $M D$

\section{Introduction}

In 1991, Page and colleagues published a report of four cases of thrombotic thrombocytopenic purpura (TTP) attributed to treatment with the platelet $\mathrm{ADP}$ receptor antagonist, ticlodipine. ${ }^{1}$ Since then, ticlodipine has been established as an immunemediated cause of TTP with an incidence of approximately $0.02-0.06 \% .^{2,3}$ Due to its unfavorable side-effect profile, the use of ticlodipine has been mostly discontinued in the United States and replaced by clopidogrel. Both agents are thienopyridine-derivatives which differ only by a carboxymethyl moiety. In spite of their structural resemblance, no case of TTP was reported in phase III trials of clopidogrel with 19,185 patients. ${ }^{4}$ However, reports of clopidogrel-associated TTP have emerged since the FDA approved the drug in 1998, including a seminal publication of eleven cases. ${ }^{5}$ Five of these cases passed an independently conducted causality assessment. ${ }^{6}$ Despite the identification of additional clopidogrel-associated cases by pharmacologic surveillance, ${ }^{7}$ skepticism remains regarding whether clopidogrel actually causes TTP. ${ }^{8}$ Here we report a case of clopidogrelassociated TTP and briefly review proposed mechanisms of drug-induced TTP.

\section{Case Report}

A 55-year-old Caucasian female presented with fever, jaundice, hematuria and painful neuropathy. Three years earlier, the patient was diagnosed with colorectal cancer which was successfully treated with resection. The patient also suffered from severe peripheral vascular disease secondary to diabetes mellitus, for which she had received an axillofemoral and aortafemoral bypass grafts several years prior. Ten years earlier, she also received a total abdominal hysterectomy with bilateral salpingo-oopherectomy for an ovarian cyst. Her medications at admission included coumadin $3 \mathrm{mg}$ qday, premarin $0.3 \mathrm{mg}$ qday, hydroxychloroquine $200 \mathrm{mg}$ qday, glucosamine, lisinopril $20 \mathrm{mg}$ qday, hydrochlorothiazide $12.5 \mathrm{mg}$ qday, cilostazol 100 mg bid, and clopidogrel $75 \mathrm{mg}$ qday. Clopidogrel had been added to her regimen within the past three months, ostensibly as adjunctive therapy for lower extremity claudication. She was an ex-smoker. She had no history of human immunodeficiency virus (HIV) or quinine exposure.

Physical exam was significant for fever $\left(38.0^{\circ} \mathrm{C}\right)$, mild jaundice and petechiae of hands and abdomen. Laboratory testing revealed abnormal hemoglobin $(5.6 \mathrm{~g} / \mathrm{dL})$, platelets $\left(8.0 \times 10^{9} /\right.$ 1), lactate dehydrogenase $(2847$ $\mathrm{IU} / \mathrm{L})$, indirect bilirubin $(3.0$ $\mathrm{mg} / \mathrm{dL})$, serum creatinine $(1.6$ $\mathrm{mg} / \mathrm{dL})$, and haptoglobin $(<0.6$ $\mu \mathrm{mol} / \mathrm{L})$. Urinalysis revealed 66 red cells per high power field. Severe schistocytosis was noted on peripheral smear (Figure 1). Direct antiglobulin testing was negative and fibrinogen levels were normal (483 mg/ dL). Serum carcinoembryonic antigen to detect recurrence of colorectal cancer was negative, and computed tomography (CT) scan of the abdomen did not show local recurrence or metastases. Blood cultures did not grow organisms. Transthoracic echocardiography showed normal cardiac function.

Although TTP was considered in her differential diagnosis, her clinicans first attributed her microangiopathic hemolytic anemia to suspected urinary tract infection. However, her status did not improve after several days with antibiotics and with consultation from hematology, the diagnosis of TTP was made at hospital day six. Serum von-willebrand factor (vWF) protease (ADAM-TS13) samples drawn during her hospital stay returned with an activity of $<5 \%$ (normal $>67 \%$ ) by fluorescence resonance energy transfer (FRET) assay, and $>$ 8.0 inhibitory units by mixing (normal $<0.4$ ), consistent with immune-mediated TTP. The patient received a total of 8 units of packed red blood cells during her stay, and therapeutic plasma exchange was performed at hospital day \#7 with 12 units of fresh frozen plasma (FFP). Twelve hours later, the

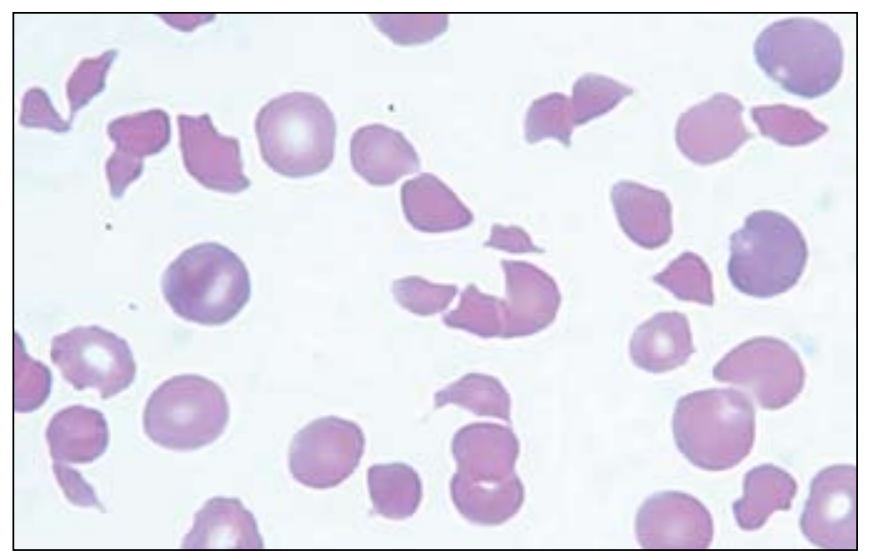

Figure 2. Peripheral blood smear at admission, 500x. Note schistocytosis, severe thrombocytopenia, and polychromasia 
patient decompensated with acute shortness of breath and hypotension, and was subsequently intubated and admitted to the intensive care unit. Shortly after transfer, she entered into pulseless electrical activity (PEA) and cardiac life support failed to resuscitate her. Her family declined autopsy. No evidence of fluid overload was seen. Based upon review of the events, it is plausible that the patient died from a massive pulmonary embolism likely secondary to her hypercoagulable state.

\section{Discussion}

With the addition of this case, there are now 38 identified instances of clopidogrel-associated thrombic microangiopathy in the literature. The time course of drug initiation is consistent with prior case series ${ }^{5}$ in which patients presented with TTP within two to three weeks after beginning a thienopyridine-derivative.

Although our patient was also receiving a synthetic derivative of quinine, no association has been made between hydroxychloroquine and TTP. In fact, thrombocytopenia alone is not reported as an adverse effect of hydroxychloroquine therapy. It is conceivable that the added hydroxychloryl group may alter the epitope of quinine ${ }^{8}$ that induces drug-dependent antibodies. Indeed, exquisite specificity has been demonstrated in other causes of antibody-mediated drug-induced thrombocytopenia, such as sulfamethoxazole ${ }^{9}$ and abciximab $^{10}$. Drug-induced autoantibodies to ADAMTS13 may exhibit similar specificity, likely accounting for the remarkably fewer cases of TTP documented with clopidogrel compared to ticlopidine.

Plasma exchange treats TTP via two plausible mechanisms: infusion and removal. Infusion of fresh donor plasma supplies the patient's circulation with the missing ADAMTS13 protease, thereby breaking down dangerous excesses of von-Willebrand factor multimers. Exchange of recipient plasma removes platelet aggregates, vWF multimers, and inhibitory autoantibodies to ADAMTS13. Plasma exchange may also filter culprit drug metabolites which may have induced TTP, such as thienopyridine derivatives. Not surprisingly, it has been observed that receipt of plasma exchange within three days of onset of clopidogrel-associated TTP results in $100 \%$ survival, vs. $27 \%$ if therapy is initiated afterwards. ${ }^{7}$ In our case, the fatality of our patient may be attributable to delayed diagnosis and consequent initiation of plasma exchange at hospital day seven.

The finding of a vWF multimer protease inhibitor in this case is consistent with prior reports of ticlopidine and clopidogrelassociated TTP. Immunoglobulins to ADAMTS13 have been found in other etiologies of TTP in which immune dysregulation is suspected, such as human immunodeficiency virus (HIV) ${ }^{10}$ and solid organ transplantation. ${ }^{11}$ Inhibitory auto-antibodies to vWF protease are almost always an IgG class, although IgA class are occasionally identified in the same sera. The presence of an ADAMTS13-activity inhibitor by mixing does not necessarily mean that an associated immunoglobulin will even be detected; nor does the presence of ADAMTS13 antibodies guarantee that a mixing test result will be positive. The latter situation is rather easily explained by observing that protease-associated antibodies need not necessarily inhibit the activity of the protease. The former situation is more problematic, but fortunately appears to be rare. In a rigorous cohort study of 35 TTP patients, Ferrari et al. $^{14}$ found that an immunoglobulin was present in $91 \%$ of patients. Two of these patients had no detectable $\operatorname{IgG} / \operatorname{IgM} / \operatorname{IgA}$

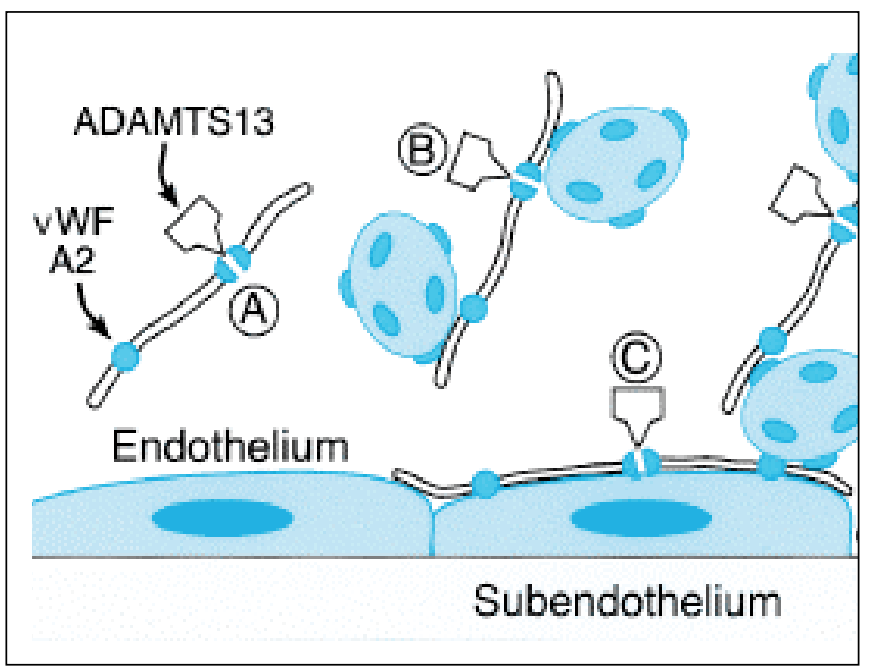

Figure 3. Endothelial cells secrete $v W F$ multimers into the circulation. The protease ADAMTS13 cleaves these multimers at the A2 domain (A). Platelets adhere to these multimers in the bloodstream (B) via the platelet glycoprotein Ib (GpIb). These multimers can also bind platelets to the endothelial surface $(\boldsymbol{C})$. Deficiency or inhibition of ADAMTS13 causes $v$ WF multimers to accumulate. Multimer accumulation results in increased platelet adherence and clot activation. This is the hypothesized mechanism of TTP. (diagram from Sadler ${ }^{15}$ )

but did have an ADAMTS13 inhibitor. Specialized clinical laboratories have also reported occasional difficulty detecting IgG with samples positive by mixing ${ }^{16}$. The inability to detect immunoglobulin in these cases may simply represent a functional limitation of the assay. Unfortunately in our case, patient blood samples were disposed of before ELISA could be performed to identify ADAMTS13 antigen and immunoglobulins. Nevertheless, the strong inhibition of ADAMTS13 in our sample ( $>8.0$ Bethesda units) makes it likely that an immune-mediated TTP was present, mostly likely secondary to clopidogrel. We also propose that specialized hematologic laboratories implement protocols to label and retain frozen blood samples of TTP for longer periods than the conventional sixty days. This practice may aid future investigators in the development of newer and more effective tests for this rare disease. 


\section{References}

1. Page Y., Tardy B., Zeni F., Comtet. C., Terrana R., Bertrand J.C. Thrombotic thrombocytopenic purpura related to ticlopidine. Lancet 1991, 337(8744):774-6.

2. Bennett CL, Weiberg PD, Rozenberg-Ben-Dror K, et al. Thrombotic thrombocytopenic purpura associated with ticlopidine: a review of 60 cases. Ann Intern Med 1998;128:541-4.

3. Steinhubl SR, Tan WA, Foody JM, Topol EJ. Incidence and clinical course of thrombotic thrombocytopenic purpura due to ticlopidine following coronary stenting. JAMA 1999; 281:806-810.

4. CAPRIE Steering Committee. A randomised, blinded, trial of clopidogrel versus aspirin in patients at risk of ischaemic events (CAPRIE). Lancet. $1996 \mathrm{Nov}$ 16;348(9038):1329-39.

5. Bennett CL, Connors JM, Carwile JM, Moake JL, Bell WR, Tarantolo SR, McCarthy LJ, Sarode R, Hatfield AJ, Feldman MD, Davidson CJ, Tsai HM. Thrombotic thrombocytopenic purpura associated with clopidogrel. N Engl J Med. 2000 Jun 15;342(24):1773-7.

6. Paradiso-Hardy FL, Papastergiou J, Lanctot KL, Cohen EA. Thrombotic thrombocytopenic purpura associated with clopidogrel: further evaluation. Can J Cardiol. 2002 Jul;18(7):771-3

7. Zakarija A, Bandarenko N, Pandey DK, et al. Clopidogrel-associated TTP: an update of pharmacovigilance efforts conducted by independent researchers, pharmaceutical suppliers, and the Food and Drug Administration. Stroke. 2004 Feb;35(2):533-7. Epub 2004 Jan 5.

8. Christie DJ; Weber RW; Mullen PC; Cook JM; Aster RH Structural features of the quinidine and quinine molecules necessary for binding of drug-induced antibodies to human platelets. J Lab Clin Med 1984 Nov;104(5):730-40.

9. Curtis BR; McFarland JG; Wu GG; Visentin GP; Aster RH. Antibodies in sulfonamide-induced immune thrombocytopenia recognize calcium-dependent epitopes on the glycoprotein IIb/IIIa complex. Blood 1994 Jul 1;84(1):176-83.

10. Curtis BR; Swyers J; Divgi A; McFarland JG; Aster RH Thrombocytopenia after second exposure to abciximab is caused by antibodies that recognize abciximabcoated platelets. Blood 2002 Mar 15;99(6):2054-9.

11. Sahud MA, Claster S, Liu L, Ero M, Harris K, Furlan M. Von Willebrand factor-cleaving protease inhibitor in a patient with human immunodeficiency syndrome-associated thrombotic thrombocytopenic purpura. Br J Haematol. 2002 Mar;116(4):909-11.

12. Nakazawa Y, Hashikura Y, Urata K, et al. Von Willebrand factor--cleaving protease activity in thrombotic microangiopathy after living donor liver transplantation: a case report. Liver Transpl. 2003 Dec;9(12):1328-33.

13. Zheng XL, Kaufman RM, Goodnough LT, Sadler JE. Effect of plasma exchange on plasma ADAMTS13 metalloprotease activity, inhibitor level, and clinical outcome in patients with idiopathic and nonidiopathic thrombotic thrombocytopenic purpura. Blood. 2004 Jun 1;103(11):4043-9. Epub 2004 Feb 24

14. Ferrari S, Scheiflinger F, Rieger M, et al. Prognostic value of anti-ADAMTS13 antibodies features (Ig isotype, titer and inhibitory effect) in a cohort of 35 adult French patients undergoing a first episode of thrombotic microangiopathy with an undetectable ADAMTS13 activity. Blood. 2006 Dec 12 [Epub ahead of print]

15. Sadler JE: A new name in thrombosis, ADAMTS13. Proc Natl Acad Sci USA 99:11552, 2002.

16. Friedman KD, Director of Hemostasis Laboratory. Personal communication regarding Imubind assay. March 2007. BloodCenter of Wisconsin, Milwaukee, Wisconsin 53201, USA.

17. National Drug Monograph Database. U.S. National Library of Medicine, Department of Health \& Human Services, 8600 Rockville Pike, Bethesda, MD 20894 